\title{
Introducción al dossier Estudios métricos de la información: abordajes teóricos, metodológicos y empíricos
}

\author{
Sandra Edith Miguel \\ Instituto de Investigaciones en Humanidades y Ciencias Sociales-IdIHCS (UNLP-CONICET), \\ Universidad Nacional de la Plata, Argentina \\ smiguel@fahce.unlp.edu.ar
}

http://orcid.org/0000-0001-9384-6838

\section{Gustavo Liberatore}

Universidad Nacional de Mar del Plata. Departamento de Ciencia de la Información, Argentina gliberat@mdp.edu.ar

http://orcid.org/0000-0002-5841-8739

En este dossier, dedicado a los Estudios Métricos de la Información (EMI), se ha convocado a especialistas iberoamericanos con el objeto de reunir diferentes aportes y miradas en este ámbito de investigación profundamente dinámico y de características particulares en términos de la transversalidad de sus contribuciones a todas las áreas del conocimiento. A pesar de ser un dominio en el que habitualmente colaboran autores de diferentes formaciones y en donde sus métodos y técnicas suelen ser aplicadas (o apropiadas) desde múltiples disciplinas, constituye en sí mismo un espacio perfectamente delimitado desde su concepción teórica y andamiaje metodológico.

En Argentina, como en muchos países de la región, los EMI han sido tradicionalmente abordados, tanto en la formación como en la investigación, desde el campo de la bibliotecología y ciencia de la información como un área destinada al análisis y estudio de aquellos fenómenos derivados de la producción, la publicación y la circulación del conocimiento. Las referencias e influencias en el desarrollo y afianzamiento de esta especialidad, en nuestro país, se encuentran asociadas a dos instituciones españolas que poseen una tradición y liderazgo en la investigación bibliométrica en Iberoamérica, que son la Universidad de Granada y la Universidad Carlos III de Madrid. Es allí donde se formaron varios de los investigadores que han consolidado a los EMI principalmente en dos de las escuelas de bibliotecología argentinas pertenecientes a la Universidad Nacional de La Plata y la Universidad Nacional de Mar del Plata. En el contexto de estas trayectorias y de las redes de contactos y actividades colaborativas con colegas de otras prestigiosas instituciones del país y de la región, el presente dossier constituye entonces un tributo al camino recorrido y un testimonio de su existencia, ya que, además es el primero de esta especialidad publicado en una revista nacional de nuestra disciplina.

La agenda de la investigación bibliométrica se encuentra hoy atravesada por desafíos relacionados con los dispositivos puestos en juego para el logro de abordajes más precisos y ajustados a situaciones y espacios concretos. El cambio de tendencia de una interpretación universal de los fenómenos observados ha dado paso, cada vez más, al diseño de indicadores que aporten resultados adecuados a los contextos en donde son aplicados. Esta característica es particularmente palpable en América Latina que, como región periférica de la ciencia mundial y territorio débilmente mapeado en términos bibliométricos, enfrenta algunas dificultades en la adopción de criterios y políticas para la medición de su capital científico desde la perspectiva de la producción y sus circuitos de circulación. En este escenario, es notoria la madurez y calidad alcanzadas en las 
investigaciones desarrolladas en el terreno de los EMI a nivel regional, que han permitido poner en debate, no sólo los modelos de análisis tradicionales, sino además los diseños metodológicos más apropiados para una mejor representación e interpretación de la realidad.

Los textos aquí reunidos recogen buena parte de las temáticas que atraviesan este ámbito. Por una parte, el enfoque latinoamericano/iberoamericano se encuentra reflejado en tres artículos que aportan resultados y reflexiones sobre las contribuciones de investigaciones bibliométricas publicadas en una revista de referencia en el campo de los EMI como Scientometrics (Miguel y Liberatore); la comparabilidad de indicadores de input/output de la ciencia iberoamericana tomando como base dos fuentes secundarias producidas por la RICYT y Scimago (Ortiz Jaureguizar-Traverso); y la colaboración científica de la universidades latinoamericanas con el mundo empresarial observada a partir del análisis de la co-autoría de la producción indizada en Scopus (Orduña Malea). Por otra parte, se destacan dos trabajos que discurren sobre cuestiones metodológicas relacionadas con la problemática de las fuentes de datos secundarias de información científica regionales e internacionales utilizadas para las investigaciones bibliométricas en términos de sus características y funcionalidades (Vuotto, Di Césare y Pallotta), y la utilización de técnicas conexionistas como la co-citación, el acoplamiento bibliográfico y la co-ocurrencia de términos en la producción científica indizada en Web of Science (Arencibia Jorge, Vega Almeida y Carrillo Calvet). Seguidamente, se incluyen dos artículos enfocados a objetos de análisis más específicos como son el estudio de la colaboración internacional en el área de la agroindustria en la Argentina (González y Chinchilla-Rodríguez) y la caracterización del autor de correspondencia (AC) en artículos publicados en otra revista de referencia en el campo como el Journal of Informetrics (Hilário, Martínez Ávila y Grácio). Por último, cierra esta presentación un estudio de caso aplicado a la identificación de líderes científicos (Ortiz-Torres y Viamonte-Garrido). 